\title{
Acute changes in liver tumour perfusion measured non-invasively with arterial spin labelling
}

\author{
S Peter Johnson ${ }^{\star 1,2,4}$, Rajiv Ramasawmy ${ }^{1,2,4}$, Adrienne E Campbell-Washburn ${ }^{2,3}$, Jack A Wells ${ }^{2}$,
} Mathew Robson ${ }^{1}$, Vineeth Rajkumar ${ }^{1}$, Mark F Lythgoe ${ }^{2,5}$, R Barbara Pedley ${ }^{1,5}$ and Simon Walker-Samuel ${ }^{2,5}$ ${ }^{1}$ UCL Cancer Institute, University College London, Paul O'Gorman Building, 72 Huntley Street, London WC1E 6DD, UK; ${ }^{2}$ UCL Centre for Advanced Biomedical Imaging, University College London, Paul O'Gorman Building, 72 Huntley Street, London WC1E $6 D D$, UK and ${ }^{3}$ Cardiovascular and Pulmonary Branch, Division of Intramural Research, National Heart, Lung and Blood Institute, National Institutes of Health, Bethesda, MD 20892, USA

Background: Non-invasive measures of tumour vascular perfusion are desirable, in order to assess response to vascular targeting (or modifying) therapies. In this study, hepatic arterial spin labelling (ASL) magnetic resonance imaging (MRI) was investigated to measure acute changes in perfusion of colorectal cancer in the liver, in response to vascular disruption therapy with OXi4503.

Methods: SW1222 and LS174T tumours were established in the liver of MF1 nu/nu mice via intrasplenic injection. Perfusion and $R_{2}^{\star}$ MRI measurements were acquired with an Agilent 9.4T horizontal bore scanner, before and at $90 \mathrm{~min}^{\mathrm{after}} 40 \mathrm{mg} \mathrm{kg}{ }^{-1} \mathrm{OXi4503}$.

Results: A significant decrease in SW1222 tumour perfusion was observed $(-43 \pm 33 \%, P<0.005)$. LS174T tumours had a significantly lower baseline level of perfusion. Intrinsic susceptibility MRI showed a significant increase in $R_{2}^{*}$ in LS174T tumours $(28 \pm 25 \%, P<0.05)$. An association was found between the change in tumour perfusion and the proximity to large vessels, with pre-treatment blood flow predictive of subsequent response. Histological evaluation confirmed the onset of necrosis and evidence of heterogeneous response between tumour deposits.

Conclusions: Hepatic ASL-MRI can detect acute response to targeted tumour vascular disruption entirely non-invasively. Hepatic ASL of liver tumours has potential for use in a clinical setting.

Angiogenesis is a key hallmark of cancer biology (Hanahan and Weinberg, 2011), and while this enables the delivery of nutrients and oxygen to meet the metabolic demands of the malignant cells, it also provides a route through which to administer therapeutic agents. Methods for measuring tumour vascular perfusion are therefore in demand, both as research tools and as a means to characterise and monitor tumours in patients. The development of non-invasive measures of vascular perfusion in tumours is particularly appropriate as the conventional radiological assessment of response (using the RECIST criteria; Eisenhauer et al,
2009) evaluates changes in tumour size, which overlooks functional changes that can occur over much shorter timescales and could be equally indicative of a successful response to the therapy.

A number of magnetic resonance imaging (MRI) techniques have been used to assess acute response to vascular targeted therapy, including contrast-enhanced MRI (Patterson et al, 2012b), intrinsic susceptibility (IS)-MRI (Robinson et al, 2005), vessel size imaging (Burrell et al, 2012; Nielsen et al, 2012) and diffusion MRI (Koh et al, 2009; Nathan et al, 2012). However, the baseline perfusion of the tumour vasculature, and the acute (i.e., within

*Correspondence: Dr SP Johnson; E-mail: peter.johnson@cancer.ucl.ac.uk

${ }^{4}$ Joint first authors.

${ }^{5}$ Joint senior authors.

Revised 26 January 2016; accepted 4 February 2016; published online 31 March 2016

(c) 2016 Cancer Research UK. All rights reserved 0007 - 0920/16

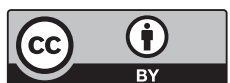


several hours of administration) assessment of response to vascular therapy could provide a better understanding of the tumour vascular microenvironment and potentially predictive indicators of drug action. Intrinsic susceptibility MRI has shown promise in the acute setting, with some indication of prognostic value in pretherapy measurements (Robinson et al, 2005). The IS-MRI can be used to quantify deoxyhaemoglobin build-up via assessment of the transverse relaxation rate $R_{2}^{*}$; however, physiological interpretation of variations in such measurements can be challenging due to its dependence on numerous physiological processes, such as vascular geometry, paramagnetic cellular debris associated with necrosis, and haemosiderin. A direct method for measurement of tumour perfusion would therefore be an improvement on IS-MRImeasured vascular haemodynamics.

We recently investigated the use of arterial spin labelling (ASL) MRI to measure the combined arterial and portal venous perfusion in both normal mouse liver and a mouse model of colorectal metastasis to the liver (Ramasawmy et al, 2014). ASL uses the flow of blood water to estimate perfusion in $\mathrm{mlg}^{-1} \mathrm{~min}^{-1}$, and thus does not require administration of a contrast agent, allowing for repeated perfusion measurements without the need to wait for a contrast agent to disperse. Perfusion can then be measured before and immediately following drug administration. Moreover, hepatic ASL offers good potential for translation onto human MRI scanners and for use in the clinic in these deep-sited tumours, particularly for patient populations that cannot be administered a contrast agent, as it has been employed in other liver conditions (Bokkers et al, 2012; Zou et al, 2014).

In the current study, we have continued our previous work by investigating the utility of ASL in the assessment of acute response to the second-generation combretastatin molecule OXi4503 (combretastatin A1 di-phosphate/CA1P), a potent vascular disrupting agent, in a mouse model of colorectal metastasis to the liver. Two human colorectal carcinoma cell lines were studied (LS174T and SW1222), with well-characterised and markedly different vascular characteristics (El et al, 2007). The aim of this research was to evaluate whether ASL can provide a direct measure of tumour perfusion for prognostic measurements both before and at an acute time point after vascular targeted therapy. As the model used created numerous metastatic tumours within the liver, a secondary aim of the study was to investigate the relationship between response to therapy and the location of tumour deposits within the liver.

\section{MATERIALS AND METHODS}

Cell lines and tissue culture. The human colorectal carcinoma cell lines SW1222 and LS174T (El et al, 2007) were cultured in Dulbecco's Modified Eagles Medium (PAA, GE Healthcare, Fairfield, CT, USA) containing $5 \mathrm{~mm}$ L-glutamine (PAA, GE Healthcare) and 10\% v/v Fetal bovine serum (Life Technologies, Carlsbad, CA, USA). Cells were grown in a $37^{\circ} \mathrm{C}$ humidified incubator at $5 \% \mathrm{CO}_{2}$.

In vivo model of liver metastasis. All animal studies were approved by the University College London Biological Services Ethical Review Committee and licensed under the UK Home Office regulations and the Guidance for the Operation of Animals (Scientific Procedures) Act 1986 (Home Office, London, UK) and United Kingdom Co-ordinating Committee on Cancer Research Guidelines for the Welfare and Use of Animals in Cancer Research (Workman et al, 2010). To establish liver tumours (Fidarova et al, 2008), MF1 nu/nu mice (female, 6-8 weeks old, 25-30 g) were anaesthetised with isoflurane in $\mathrm{O}_{2}$ at a concentration of $4 \%$ for induction, followed by $1.5 \%$ for maintenance. A subcutaneous injection of $0.1 \mathrm{mg} \mathrm{kg}^{-1}$ buprenorphine (Vetergesic, Reckitt
Benckiser, UK) was administered and the flank of the animal sterilised with clorhexidine solution. A laparotic incision of approximately $1 \mathrm{~cm}$ was then made above the location of the spleen and the organ exteriorised for injection. SW1222 $(n=6)$ or LS174T $(n=6)$ cells were injected intrasplenically at a concentration of $1 \times 10^{6}$ cells in $100 \mu$ l of serum-free medium and allowed to wash through to the liver for $5 \mathrm{~min}$, followed by splenectomy to avoid the development of tumours at the site of injection. The laparotic incision was sutured (Ethicon, Johnson \& Johnson, New Brunswick, NJ, USA) and closed with wound clips (Autoclip system; Harvard Apparatus, Cambridge, UK).

Magnetic resonance imaging. All MRI was performed on a 9.4-T Agilent VNMRS scanner (Agilent Technologies, Santa Clara, CA, USA) with a 39-mm birdcage coil (RAPID Biomed, Rimpar, Germany). Mice were anaesthetised with isoflurane in $\mathrm{O}_{2}$ at a concentration of $4 \%$ for induction followed by $1-2 \%$ for maintenance. Mice were then positioned in the centre of the bore of the magnet, maintained at $37^{\circ} \mathrm{C}$ with heated water pipes, and monitored using respiratory bellows and a rectal temperature probe (SA Instruments, Stony Brook, NY, USA).

Assessment of model development with morphological MRI. A respiratory-gated, high-resolution, $T_{2}$-weighted, fast spin echo (FSE) sequence was used to determine tumour burden at 3, 4 and 5 weeks post implantation and later used to classify the location of tumour deposits. Sequence parameters included: field of view $(\mathrm{FOV})=35 \times 35 \mathrm{~mm}^{2}, \quad$ matrix size $=256 \times 256, \quad \mathrm{TR}=1500 \mathrm{~ms}$, echo train length $=4$, effective $\mathrm{TE}=26 \mathrm{~ms}, 40 \times 0.5 \mathrm{~mm}$ contiguous slices to cover the entire liver, and four averages.

Voxels corresponding to liver and tumour tissue, which appeared hyper-intense relative to normal liver, were manually segmented using Amira (Visualisation Sciences Group, Hillsboro, OR, USA). Tumour locations were manually assessed on highresolution $T_{2}$ weighted images and divided into three categories: (a) peripheral, (b) near small, and (c) near large vessels. The limit to the tumours' proximity to major vasculature was defined as 3-4 voxels $(0.5 \mathrm{~mm})$. Venous and arterial blood vessels appear hypointense on the $T_{2}$-weighted image and small vessels were categorised by a cross-sectional area less than $0.1 \mathrm{~mm}^{2}$ (approximately 7-8 voxels in diameter).

Functional assessment of liver tumour response to vascular disruption. Assessment of vascular perfusion change within liver tumours following therapy with the VDA OXi4503 was performed at 5 weeks post surgery. All six SW1222 tumour-bearing mice were dosed with $40 \mathrm{mg} \mathrm{kg}^{-1}$ of OXi4503, with normal liver acting as an inherent internal control in order to minimise animal usage and to provide relevant indication of functional change within the tumours. Only three out of six LS174T models developed distinct liver tumours; therefore, only these were put forward for VDA therapy, again using internal controls of normal-appearing liver tissue. Based on results from these initial experiments, a further six SW1222 models were set up as external standalone controls, five of which produced liver metastases. These mice were set up and imaged exactly as the treated experimental groups albeit dosed with a saline sham dose instead of OXi4503.

A primed intravenous (i.v.) line was positioned in the tail for administration of OXi4503. Following morphological scanning, an axial slice through the liver was identified for ASL and IS-MRI scans. Baseline perfusion and $R_{2}{ }^{*}$ measurements were performed before administering $40 \mathrm{mg} \mathrm{kg}^{-1}$ OXi4503 (4 $\mathrm{mg} \mathrm{ml}^{-1}$ stock solution injected at $10 \mathrm{ml} \mathrm{kg}^{-1}$ of body weight) via the i.v. line. $R_{2}{ }^{*}$ measurements were acquired dynamically up to $90 \mathrm{~min}$ post dose, and were followed by a second perfusion acquisition. The time point of $90 \mathrm{~min}$ was used as previous in-house experiments in subcutaneous tumours showed $R_{2}{ }^{*}$ increases after OXi4503 dosing within this time frame. 
Arterial spin labelling. Arterial spin labelling data were acquired using a single-slice respiratory triggered, Look-Locker flowsensitive alternating inversion recovery (FAIR) with a segmented gradient-echo readout (Campbell-Washburn et al, 2013). Sequence parameters included: slice thickness $=1 \mathrm{~mm}, \mathrm{FOV}=30 \times 30 \mathrm{~mm}$, matrix size $=128 \times 128$, echo time $(\mathrm{TE})=1.18 \mathrm{~ms}$, inversion time spacing $=110 \mathrm{~ms}$, Look-Locker readout pulse spacing $2.3 \mathrm{~ms}$, flip angle $=8^{\circ}$. Sequence repetition time: $13 \mathrm{~s}, 50$ inversion recovery readouts, 4 lines per segmented acquisition. A 6-mm localised selective inversion was followed by a global inversion, with the whole scan lasting around $14 \mathrm{~min}$.

Total liver perfusion was quantified using methods previously described (Ramasawmy et al, 2014); briefly, the data were quantified with the Belle model (Belle et al, 1998), alongside an intravascular capillary blood longitudinal relaxation time that had been previously measured in the ventricular blood pool of the mouse heart at $1.9 \mathrm{~s}$ (Campbell-Washburn et al, 2012). A bloodtissue partition coefficient of $0.95 \mathrm{mlg}^{-1}$ was taken from ${ }^{85}$ Krypton gas-clearance measurements previously reported (Rice et al, 1977), and this constant was used for both liver and tumour regions. A three-parameter fit of the inversion recovery, corrected for the Look-Locker signal saturation (Deichmann, 1992), was used for longitudinal relaxation time $\left(T_{1}\right)$ quantification following retrospective gating analysis.

Perfusion values were calculated in regions of interest (ROIs) corresponding to either tumour or normal tissue in the single slice acquired in the ASL acquisition. Mean perfusion values were estimated in each ROI.

Intrinsic susceptibility MRI. Values of the transverse relaxation rate $\left(R_{2}^{*}\right)$ were estimated from a respiratory-gated multi-echo, multi-slice gradient-echo sequence (MGEMS). Sequence parameters included: 8 echoes $\left(\mathrm{TE}_{1}=2 \mathrm{~ms}\right.$, echo spacing $=2 \mathrm{~ms}$ ), $\mathrm{TR}=280 \mathrm{~ms}$, matrix size $=128 \times 128, \quad \mathrm{FOV}=30 \times 30 \mathrm{~mm}^{2}, \quad 15$ slices, and slice thickness $=1 \mathrm{~mm} . R_{2}{ }^{*}$ was estimated using a maximum likelihood approach that took into account the Rician distribution of the data (Walker-Samuel et al, 2010). Regions of interest corresponding to individual tumours were manually defined on all slices within the liver, and the average $R_{2}{ }^{*}$ value estimated in each tumour.

A
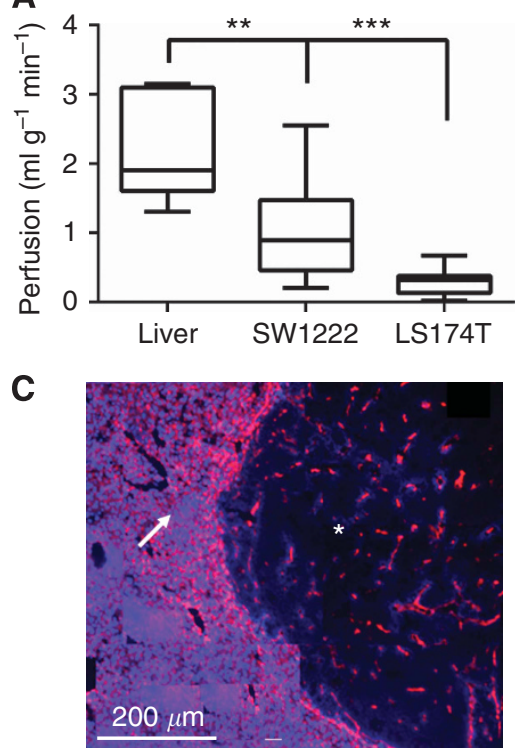

Wilcoxon tests were performed on metastases and internal controls of normal-appearing liver, before and after in situ dose for both perfusion and $R_{2}{ }^{*}$ measurements.

Histological assessment. Tumours were excised at $24 \mathrm{~h}$ post VDA administration for histological assessment. Animals were administered $12.5 \mathrm{mg} \mathrm{kg}^{-1}$ of the perfusion marker Hoechst 33342 i.v. $1 \mathrm{~min}$ before culling. Livers were excised and snap frozen in isopentane/ liquid nitrogen, before sectioning on a cryostat at $10 \mu \mathrm{m}$ thickness. Immunohistochemical (IHC) staining for the blood vessel marker CD31 was performed before imaging on a fluorescence microscope (AxioImager, Zeiss, Oberkochen, Germany). Haematoxylin and eosin staining for basic cell morphology was performed and imaged on a brightfield microscope (AxioSkop, Zeiss).

\section{RESULTS}

SW1222 vs LS174T perfusion measurements. Initial measurements of perfusion and $R_{2}{ }^{*}$, for both SW1222 and LS174T metastases and normal surrounding liver tissue (at 5 weeks post implantation), can be seen in Figure 1. Segmentation of the metastases was performed based on $T_{1 \text {,selective maps, as it was }}$ observed that the tumour $T_{1, \text { selective }}$ was significantly greater $(P<0.0001$, Kruskal-Wallis ANOVA) than that of the liver and major vasculature for both cell lines: $T_{1, \text { liver }} 1.34 \pm 0.07 \mathrm{~s}$ (mean \pm s.d., data combined from both cell lines), $T_{1 \text {,Portal vein }}$ $0.29 \pm 0.21 \mathrm{~s}$ (both cell lines), $T_{1, \mathrm{SW} 122} 2.15 \pm 0.11 \mathrm{~s} \quad(n=6)$, and $T_{1, \mathrm{LS} 174 \mathrm{~T}} 2.49 \pm 0.13 \mathrm{~s}(n=3)$.

Perfusion measurements from these metastases showed a significant difference $(P<0.0001$, Kruskal-Wallis ANOVA) between the liver, SW1222 and LS174T metastases: $P_{\text {liver }}$ $2.32 \pm 0.34 \mathrm{ml} \mathrm{g}^{-1} \mathrm{~min}^{-1}$ (mean $\left.\pm \mathrm{std}\right), P_{\mathrm{SW} 1222} 1.03 \pm 0.72 \mathrm{mlg}^{-1}$ $\min ^{-1}, P_{\mathrm{LS} 174 \mathrm{~T}} 0.28 \pm 0.16 \mathrm{ml} \mathrm{g}^{-1} \mathrm{~min}^{-1}$ (Figure 1A). This lower level of perfusion in tumours, compared with normal liver, is consistent with qualitative fluorescence microscopy imaging of perfusion seen in Figure 1C and D, where the presence of the perfusion marker Hoechst (blue) is lower in the tumour tissue ${ }^{*}$ ) for both SW1222 (Figure 1C) and LS174T (Figure 1D) compared with normal surrounding liver parenchyma (arrow). Blood vessels,

\section{B}

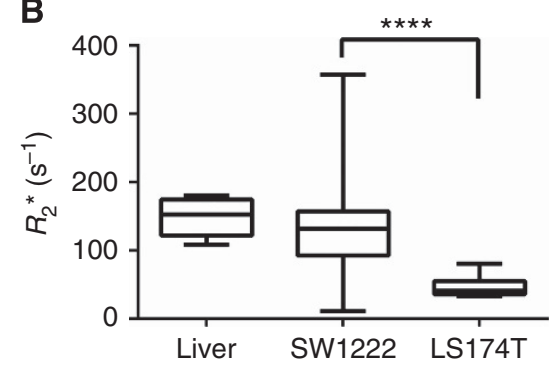

D

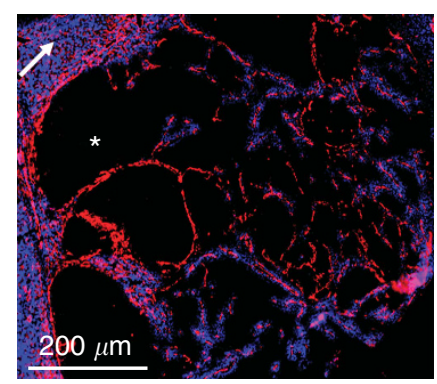

Figure 1. Baseline perfusion of tumour metastases. Average baseline estimates of blood perfusion from ASL (A) and $R_{2}{ }^{*}$ from IS-MRI (B) in normal-

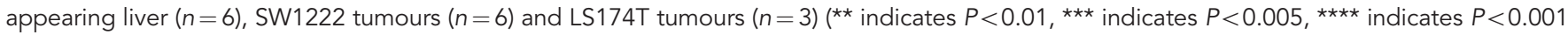
significant differences). Fluorescence microscopy of blood vessels (CD31, red) and perfusion (Hoechst 33342, blue) in SW1222 tumours (C) and LS174T tumours (D). Arrows indicate areas of normal liver tissue and stars indicate areas of tumour tissue. 
marked by CD31 in red, show a decreased abundance, penetration, and organisation within tumour tissue compared with normal liver.

A difference between SW1222 and LS174T tumours is also observable, with fewer blood vessels and perfusion in the LS174T tumours, further corroborating the obtained ASL measurements. This may contribute to the observed difference in $R_{2}{ }^{*}$ (Figure 1B), where LS174T tumours have a significantly slower value than SW1222 tumours and normal liver. A significant difference $(P<0.0001$, Kruskal-Wallis ANOVA) was measured between average $R_{2}{ }^{*}$ estimates in normal-appearing liver, LS174T tumours and SW1222 tumours: $R_{2}{ }^{*}$ liver $=148 \pm 010 \mathrm{~s}^{-1}, R_{2}{ }^{*}$ SW1222 $=144 \pm 17 \mathrm{~s}^{-1}$, and $R_{2}{ }_{\mathrm{LS} 174 \mathrm{~T}}=47 \pm 4 \mathrm{~s}^{-1}$.

Functional response of the tumour vasculature to disruption with OXi4503. Before OXi4503 delivery, tumours displayed variable baseline measurements of perfusion and $R_{2}{ }^{*}$, with a heterogeneous spatial distribution for each parameter within each tumour. Following treatment with OXi4503, a heterogeneous response was observed in both tumour types by ASL and IS-MRI techniques, as can be seen in the example maps shown in Figure 2A. Absolute and relative changes from baseline in perfusion and $R_{2}{ }^{*}$ measurements across groups are shown in Table 1, with normal appearing liver from the same animals included as an internal control; a significant decrease in perfusion was detected in SW1222 metastases for both treated and saline control groups. The decrease in perfusion of the saline control group is not fully understood, though actual perfusion change is small, it is potentially due to minor dehydration caused by prolonged imaging (Ramasawmy et al, 2014). No significant change was measured in treated LS174T tumours, probably due to their low levels of baseline perfusion. Perfusion in normalappearing liver tissue was varied, although it decreased nonsignificantly across the groups.

For change in $R_{2}^{*}$ the only significant change measured was within the VDA-treated LS174T tumours, with no significant changes observed in the SW1222 tumours (treated and control) or normal-appearing liver. Histological assessment of tumour metastases viability by $\mathrm{H} \& \mathrm{E}$ staining shows that saline-dosed control SW1222 tumours (Figure 2B) are highly nucleated and viable, with little areas of necrosis, while both OXi4503-treated SW1222 (Figure 2C) and LS174T (Figure 2D) tumours have large central necrosis with a small rim of surviving tumour cells surrounding the metastasis. No change was observed in the surrounding normal liver tissue.

Relationship between baseline and post-therapy parameter estimates. Figure 3 displays the results of an analysis comparing the change in perfusion and $R_{2}{ }^{*}$ values at $90 \mathrm{~min}$ following Oxi4503 administration, with values measured at baseline. A significant, negative correlation $\left(R^{2}=0.76, P<0.001\right.$ Pearson's $R$ ) was found between the change in tumour perfusion and the value at baseline in SW1222 tumours (Figure 3A). However, no corresponding correlation was observed between baseline $R_{2}{ }^{*}$ and subsequent change in IS $(P>0.1$, see Figure $3 \mathrm{C})$. For LS174T tumours, no significant correlation $(P>0.1)$ was measured between the 90-min changes in perfusion and baseline perfusion (Figure 3B), nor between baseline and $90 \mathrm{~min}$ changes in $R_{2}{ }^{*}$ (Figure 3D).

Relationship between response to OXi4503 and tumour location. OXi4503-treated SW1222 tumours were categorised to evaluate the effect of location within the liver and their subsequent therapeutic response. Figure $4 \mathrm{~A}$ shows a manually-segmented three-dimensional rendering of a mouse liver from $T_{2}$-weighted MRI data, in which the liver parenchyma can be seen in semitransparent red, the major vasculature in purple. Tumours have been coloured as classified by their proximity to large vessels ( $>8$ voxels, red), small vessels ( $<8$ voxels, green) and distal ( $>4$ voxels, blue) to vasculature - typically located at the periphery of the liver lobe (blue).

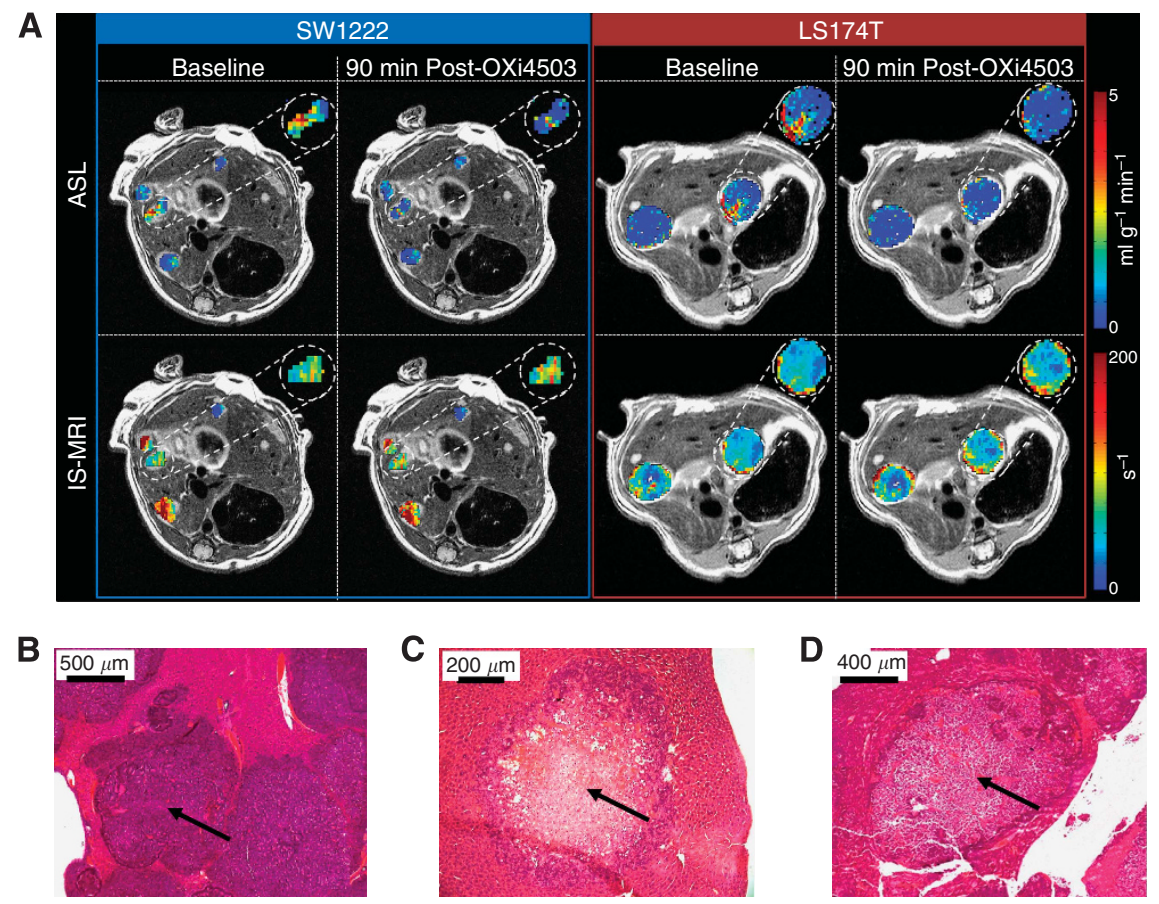

Figure 2. Functional response of the tumour vasculature to disruption with OXi4503. Example high-resolution, axial images (A) with perfusion (top row) and $R_{2}{ }^{*}$ (bottom row) maps overlaid on tumour ROIs (SW1222 on the left, and LS174T on the right, pre- and post OXi4503 therapy). A heterogeneous spatial distribution can be seen in both parameters, both within individual tumour deposits and across different tumours within the same liver. Histological staining by H\&E is shown for saline control (B), and OXi4503-dosed SW1222 (C) and LS174T (D) tumours. The arrows highlight the centre of liver tumour deposits ( $\mathbf{B}$, viable and $\mathbf{C}$ and $\mathbf{D}$, necrotic). 
Table 1. Absolute and percentage changes (mean \pm s.d.) in perfusion and $R_{2}{ }^{*}$ for $\mathbf{S W} 1222$ metastases treated with $0 X i 4503$

$(n=6)$, LS174T metastases treated with OXi4503 $(n=3)$ and SW1222 metastases given saline control $(n=5)$

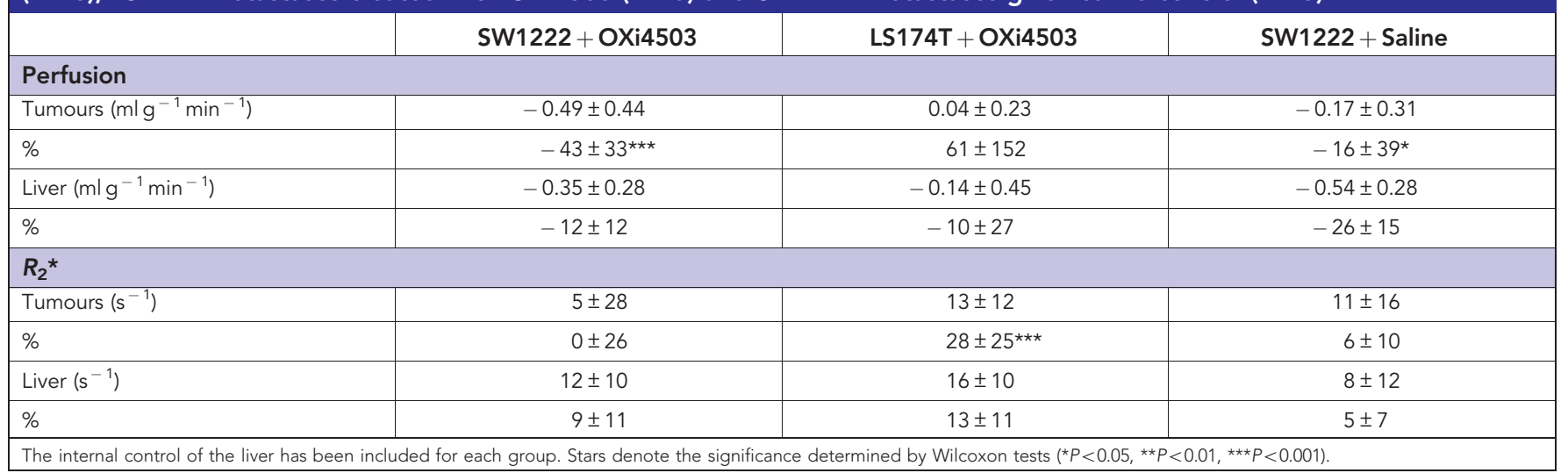
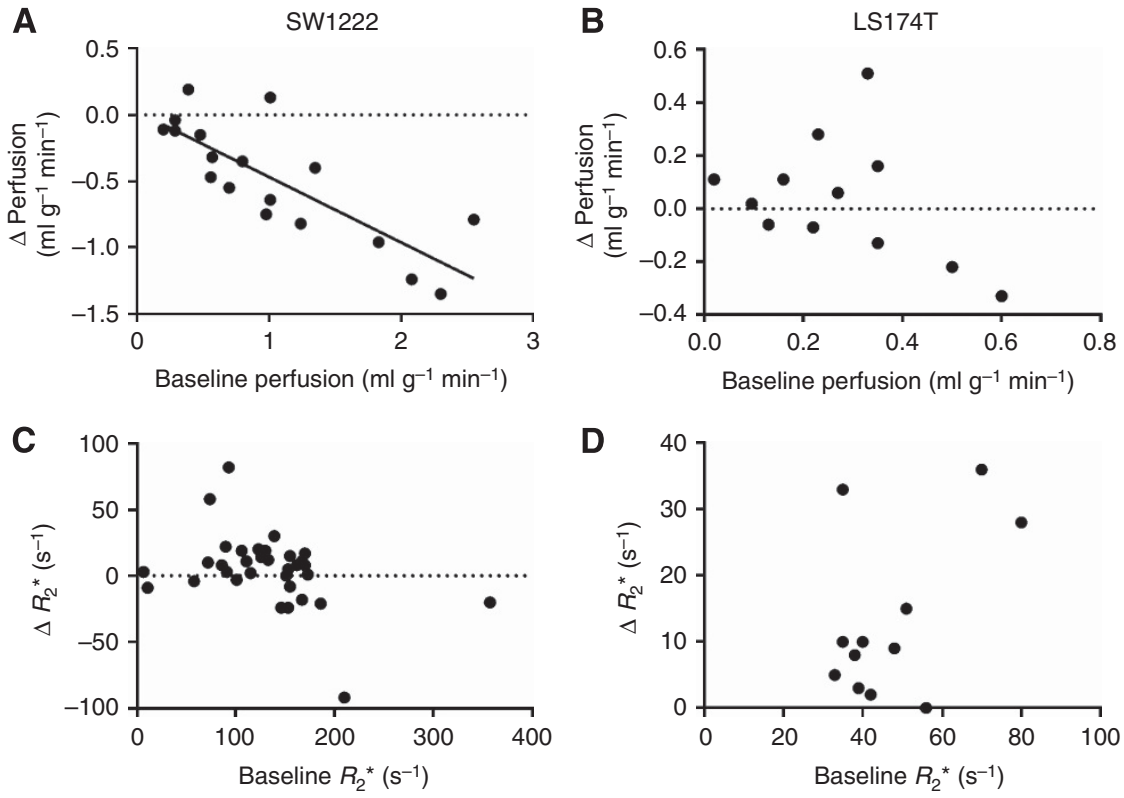

Figure 3. Correlation of baseline and post-OXi4503 measurements for perfusion (top row) and $R_{2}$ * (bottom row). Graphs show the change in the value of each parameter in individual tumour deposits vs the baseline value. The change in perfusion measured in SW1222 tumours (A) displayed a significant negative correlation with baseline measurements $\left(R^{2}=0.76, P<0.001\right.$ Pearson's $\left.R\right)$. Better-perfused tumours at baseline exhibited a greater loss of perfusion following OXi4503 administration. LS174T tumour perfusion (B) was low at baseline, and no similar trend was found. For both SW1222 (C) and LS174T (D) tumours, the $R_{2}{ }^{*}$ baseline data showed no correlation with baseline values.

Figure $4 \mathrm{~B}$ shows the change in perfusion (red, green and blue outline) and $R_{2}^{*}$ (solid red, green and blue) at 90 min post OXi4503 when liver tumours were grouped according to their location (LV: large vessels, SV: small vessels, and P: peripheral). Tumours close to major vasculature were better perfused at baseline $(P<0.05$, Kruskal-Wallis ANOVA): $1.5 \pm 0.8 \mathrm{mlg}^{-1} \mathrm{~min}^{-1}$ (mean \pm standard deviation), $1.3 \pm 0.7 \mathrm{mlg}^{-1} \mathrm{~min}^{-1}, 0.6 \pm 0.3 \mathrm{ml} \mathrm{g}^{-1} \mathrm{~min}^{-1}$ for locations LV, SV, and P, respectively. No significant differences in perfusion reduction were measured between the groups $(P>0.1$, Kruskal-Wallis ANOVA), though the data suggest that the largest perfusion decreases can be observed in metastases proximal to vasculature. The most consistent mean decrease in perfusion was observed close to large vessels ( $-62 \pm 19 \%$, mean \pm standard deviation), whereas a more varied decrease was seen in tumours near smaller vessels ( $-36 \pm 51 \%$ ). Tumours in the periphery of the liver lobe displayed a smaller but highly variable response in perfusion ( $-32 \pm 25 \%$ ).

No significant differences $(P>0.05$, Kruskal-Wallis ANOVA) were measured between baseline $R_{2}{ }^{*}$ measurements, which were $110 \pm 50 \mathrm{~s}^{-1}, 270 \pm 140 \mathrm{~ms}^{-1}$, and $210 \pm 110 \mathrm{~s}^{-1}$ for locations LV,
SV, and P, respectively. No significant differences were measured in $R_{2}{ }^{*}$ response post-OXi3403 as grouped by location $(P>0.15$, Kruskal-Wallis). Metastases in all locations behaved inconsistently for $R_{2}{ }^{*}$ (large vessel $16 \pm 26 \%$ (mean \pm standard deviation), small vessels $-13 \pm 29 \%$, and periphery $-4 \pm 20 \%$ ). Histological assessment (Figure 4C), while not quantitative, provided some evidence that tumour deposits located in close proximity could have different responses to treatment, potentially moderated by their distance from major blood vessels.

\section{DISCUSSION}

The aim of this study was to evaluate the response of a model of liver metastasis to vascular disruption using hepatic ASL-MRI to measure tumour perfusion, alongside IS-MRI as a measure of deoxyhaemoglobin accumulation. This is a novel application of ASL, demonstrating the first use of hepatic ASL-MRI to assess response to vascular targeting therapy within the liver. The results 
A

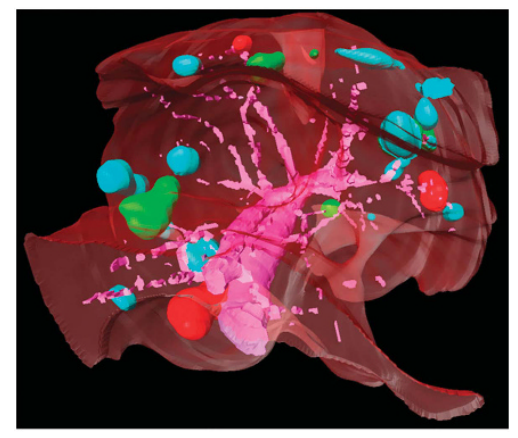

B

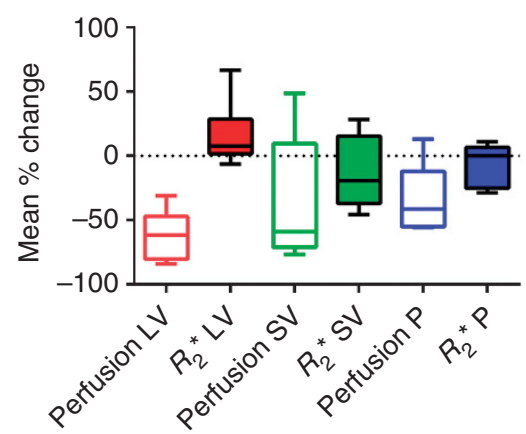

C

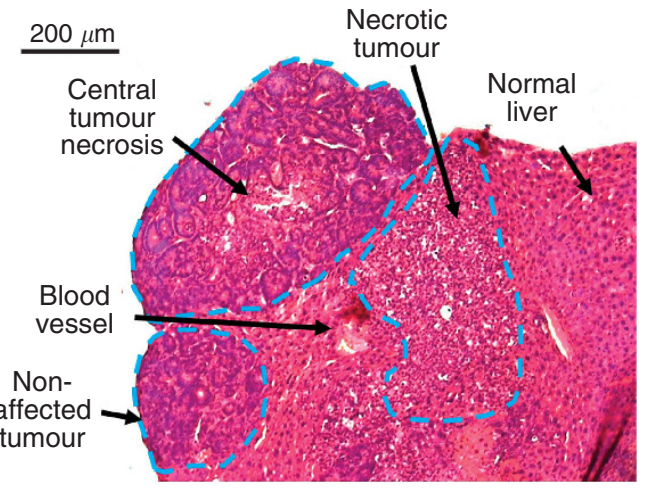

Figure 4. The dependency of tumour location within the liver and the magnitude of perfusion changes. Analysis of SW1222 tumours following dosing with OXi4503. (A) A three-dimensional visualisation of an example mouse liver at week 5 post implantation, showing liver parenchyma (semi-transparent red), and major vasculature (purple). Tumours have been coloured according to their proximity to either a large vessel (>0.1 $\mathrm{mm}^{2}$, red), small vessel (green) or no visible vessel (blue). (B) The percentage change in perfusion and $R_{2}{ }^{*}$ at 90 min following administration of $40 \mathrm{mg} \mathrm{kg}^{-1}$ of OXi4503, categorised according to proximity to vessels and location within the liver. Large perfusion decreases were observed in tumours close to either large (LV) or small (SV) blood vessels, although tumours near smaller vessels demonstrated a more variable response (as evidenced by larger error bars). Tumours at the periphery (P) of the liver did not display a significant change in perfusion. No significant changes were measured in $R_{2}{ }^{*}$ in any of the liver locations. (C) Example H\&E slice of liver containing three different tumours located at a range of position to a blood vessel, demonstrating a heterogeneous response to OXi4503 within the same liver.

presented in this paper are consistent with previous research using ultrasound imaging in subcutaneous tumours imaged before OXi4503 treatment and $30 \mathrm{~min}$ after (Mason et al, 2011), previous acute studies of a different VDA (ZD6126) (Robinson et al, 2005), the proposed vascular targeting action of the agent (Siemann, 2011), and relate well to ex vivo histological data from a similar VDA (CA4P) used in SW1222 subcutaneous tumours (El-Emir et al, 2005). The results are also consistent with other studies investigating OXi4503 (CA1P) in particular, which has been shown to significantly reduce tumour blood vessel perfusion within 30 min of dosing with a $90 \%$ reduction observed at $4 \mathrm{~h}$, albeit in a subcutaneous setting of different tumour types (Salmon and Siemann, 2006), using ex vivo histology and in vivo dynamic contrast enhanced MRI. OXi4503 has also been used in an intravital setting through use of a window chamber model of breast cancer (Wankhede et al, 2010), showing tumour vascular structural collapse from the earliest time-point imaged of $2 \mathrm{~h}$.

The use of hepatic ASL in the present study allowed novel insight into the dynamics of response to vascular disruption in an orthotopic setting and exogenous contrast label free, based on tumour pathophysiology and location within the liver. These observations could potentially inform future prognostic markers of response, and are discussed below.

Hepatic ASL is able to monitor differences in response to vascular disruption. Given that the extent of vascularisation of LS174T tumours is known to be low and much less extensive than the SW1222 tumours (Kalber et al, 2008; Folarin et al, 2010), which in turn are less well perfused than normal tissue (Figure 1), the perfusion measured in these different tissues reflect this. A significant reduction in perfusion was observed at $90 \mathrm{~min}$ following administration of OXi4503 in SW1222 tumours, while perfusion changes in LS174T tumours were highly varied, due to the low baseline values (Figure 2A; Table 1). A low initial level of perfusion is a limitation of the ASL technique's sensitivity, as further decreases may be below the detection threshold of the technique, though this would indicate that there was minimal vasculature to target. A reduction in perfusion of SW1222 tumours was also noted after saline control dose, albeit not as pronounced; the physiological mechanism behind this change is not known; however, it does highlight the sensitivity of the method used.
A significant change in $R_{2}{ }^{*}$ measurements was only observed in post-treatment LS174T tumours, indicating that a build-up of deoxyhaemoglobin and subsequent change in $R_{2}{ }^{*}$ may act as a complimentary biomarker when tumour perfusion is minimal. Histological assessment of tumour deposits showed viable tissue for saline controls (Figure 2B), and centrally necrotic deposits for OXi4503-treated SW1222 (Figure 2C) and LS174T (Figure 2D) tumour tissue.

Relationship between baseline and post-OXi4503 perfusion measurements. We observed that baseline perfusion in SW1222 tumour deposits was significantly correlated with the subsequent decrease in perfusion at $90 \mathrm{~min}$ following OXi4503 administration (Figure 3). Such acute, non-invasive measurements of perfusion changes are not generally possible, as the majority of other approaches require a contrast agent to be administered (Lee et al, 2014), and disperse, before follow-up measurements can be taken. One of the few examples of rapid tumour imaging of perfusion without the need for extended periods of contrast agent dispersal is the use of microbubbles in ultrasound, which are dispersed within minutes following administration (Mason et al, 2011). Ultrasound has also been shown to be capable of imaging vascular disruption over a similar time course without contrast agent, through the use of Colour-Doppler technique (Hadimani et al, 2013). However, this was in a superficial subcutaneous tumour siting; in this study, we have shown that ASL-MRI can correlate pre- and posttreatment perfusion in tumour sited deeply within an organ.

Therefore, ASL-MRI could be a predictor of acute VDA efficacy, with potential use as a diagnostic tool for patient stratification and treatment planning. $R_{2}{ }^{*}$, for example, has been used clinically to assess response to OXi4503, though no acute response $(<3 \mathrm{~h})$ was seen (Patterson et al, 2012a). The potential to highlight those metastases that may respond positively to treatment $v s$ those that will not, based on baseline perfusion, could spare patients from unnecessary treatment. Targeting of different tumour pathophysiologies often requires combination approaches to treatment for adequate response, especially with regard to VDAs (Pedley et al, 2002), so knowledge obtained from a biomarker of tumour perfusion could provide a deeper insight into the mechanics of tumour response to therapy in vivo.

Robinson et al (2005) found baseline $R_{2}{ }^{*}$ to be predictive of subsequent response, although this was evaluated in different cell 
lines and using a different VDA, and in a different tumour model. No equivalent result was found in this study, which could be due to the difficulty in measuring $R_{2}{ }^{*}$ in liver, particularly as shimming of the $B_{0}$ field can be challenging due to susceptibility artefacts propagating from surrounding lungs and gut, in addition to respiratory and peristaltic motion artefacts which can be difficult to control.

Heterogeneity of response to therapy based on tumour location. Vascular disrupting agents are often said to be more effective against larger tumours (Landuyt et al, 2000; Liu et al, 2015); however, in this study we found no obvious dependence of change in perfusion based on size. Certain tumour deposits, however, based on histological assessment, were not affected by OXi4503 at $24 \mathrm{~h}$ post treatment (Figure $4 \mathrm{C}$ ). As this was an unexpected result, it was not possible to retrospectively link affected tumours to perfusion measurements. The noted heterogeneity could be a function of the time course of tumour necrosis, with those tumour deposits that had not initially responded proceeding to do so at a later point in time.

However, a potential factor influencing heterogeneous response to OXi4503 treatment is the location of the tumour deposits within the liver. The pharmacokinetics of drug delivery to the tumours might be affected by systemic perfusion within the liver. By categorising location within the liver relative to major blood vessels, in an attempt to account for different degrees of perfusion, we found that the reduction in perfusion was greatest and most consistent in metastases close to large vessels (measured to have a high level of baseline perfusion), similar but less consistent in metastases close to smaller vessels (considered to have a medium level of baseline perfusion), and was smallest in those at the periphery of the liver (considered to be the least perfused tumours) (Figure 4B).

Although we were unable to use histology data to directly link this reduction in perfusion to direct action of vascular disruption, it is still indicative of differential responses of metastases to the VDA, as also demonstrated by the range of efficacy seen in neighbouring tumour deposits by IHC (Figure 4C). This suggests that the pharmacokinetics of drug action may be influenced by microenvironmental factors such as tumour perfusion. This range of responses between metastases dependent upon location and extent of growth has been previously observed with the use of radiolabelled antibodies (Siemann, 2011), which lends support to our observations.

A limitation of this study was the use of a single-slice ASL acquisition sequence. This permitted only partial coverage of the liver; and hence, a number of metastases were omitted from analysis; longitudinal measurements were also difficult as the replication of exact image slice positions between sessions was challenging. Since completion of this study, a multi-slice adaption of the Look-Locker FAIR ASL sequence has been developed (Campbell-Washburn et al, 2013). Utilising the vessel-selective pseudo-continuous ASL sequences (Dai et al, 2008) could provide a direct method for separating the arterial and venous contributions to the liver blood supply, from which response biomarkers such as the hepatic perfusion index, a measure of the relative contribution of the two supplies in individual metastases, could be evaluated to further probe tumour pathophysiology (Cuenod et al, 2001; Schalkx et al, 2014).

\section{CONCLUSION}

This study has demonstrated the use of hepatic ASL-MRI to detect acute response to the VDA OXi4503 in a mouse model of colorectal carcinoma metastasis to the liver. Significant decreases in SW1222 tumour (but not normal liver) perfusion were observed at
90 min following administration, and baseline perfusion measurements were found to be predictive of subsequent response. Moreover, response to the drug was heterogeneous between tumour deposits, with the siting of tumour deposits in the liver relative to major vessels found to have a role. Given the ability of hepatic ASL to measure acute changes in liver perfusion entirely noninvasively, it has the potential to be used in a therapeutic clinical setting.

\section{ACKNOWLEDGEMENTS}

We would like to thank OXiGENE (CA, USA) for kindly supplying the vascular disrupting agent OXi4503. This work was carried out as part of King's College London and UCL Comprehensive Cancer Imaging Centre, Cancer Research UK \& Engineering and Physical Sciences Research Council, in association with the Medical Research Council (MRC), Department of Health and British Heart Foundation (England) (C1519/A10331, C1519/A16463). RR receives funding from the MRC (MRC 1028351). RBP receives funding from Medical Research Funding DPFS grant (MRC G100149). MFL receives funding from MRC (MR/J013110/1); the National Centre for the Replacement, Reduction and Refinement of Animal in Research (NC3Rs); UK Regenerative Medicine Platform Safety Hub (MRC: MR/K026739/1). SW-S and SPJ receive funding from the Wellcome Trust (WT100247MA). JW receives funding from the Medical Research Council (Grant code: 42450).

\section{CONFLICT OF INTEREST}

The authors declare no conflict of interest.

\section{REFERENCES}

Belle V, Kahler E, Waller C, Rommel E, Voll S, Hiller KH, Bauer WR, Haase A (1998) In vivo quantitative mapping of cardiac perfusion in rats using a noninvasive MR spin-labeling method. J Magn Reson Imaging 8(6): 1240-1245.

Bokkers RP, Hernandez DA, Merino JG, Mirasol RV, van Osch MJ, Hendrikse J, Warach S, Latour LL (2012) Whole-brain arterial spin labeling perfusion MRI in patients with acute stroke. Stroke 43(5): $1290-1294$.

Burrell JS, Bradley RS, Walker-Samuel S, Jamin Y, Baker LC, Boult JK, Withers PJ, Halliday J, Waterton JC, Robinson SP (2012) MRI measurements of vessel calibre in tumour xenografts: comparison with vascular corrosion casting. Microvasc Res 84(3): 323-329.

Campbell-Washburn AE, Price AN, Wells JA, Thomas DL, Ordidge RJ, Lythgoe MF (2013) Cardiac arterial spin labeling using segmented ECG-gated Look-Locker FAIR: variability and repeatability in preclinical studies. Magn Reson Med 69(1): 238-247.

Campbell-Washburn AE, Zhang H, Siow BM, Price AN, Lythgoe MF, Ordidge RJ, Thomas DL (2012) Multislice cardiac arterial spin labeling using improved myocardial perfusion quantification with simultaneously measured blood pool input function. Magn Reson Med 70(4): 1125-1136.

Cuenod C, Leconte I, Siauve N, Resten A, Dromain C, Poulet B, Frouin F, Clement O, Frija G (2001) Early changes in liver perfusion caused by occult metastases in rats: detection with quantitative CT. Radiology 218(2): 556-561.

Dai W, Garcia D, de BC, Alsop DC (2008) Continuous flow-driven inversion for arterial spin labeling using pulsed radio frequency and gradient fields. Magn Reson Med 60(6): 1488-1497.

Deichmann R, Haase A (1992) Quantification of T1 values by SNAPSHOTFLASH NMR imaging. J Magn Reson 96: 608-612.

Eisenhauer EA, Therasse P, Bogaerts J, Schwartz LH, Sargent D, Ford R, Dancey J, Arbuck S, Gwyther S, Mooney M, Rubinstein L, Shankar L, Dodd L, Kaplan R, Lacombe D, Verweij J (2009) New response evaluation 
criteria in solid tumours: Revised RECIST guideline (version 1.1). Eur J Cancer 45(2): 228-247.

El EE, Qureshi U, Dearling JL, Boxer GM, Clatworthy I, Folarin AA, Robson MP, Nagl S, Konerding MA, Pedley RB (2007) Predicting response to radioimmunotherapy from the tumor microenvironment of colorectal carcinomas. Cancer Res 67(24): 11896-11905.

El-Emir E, Boxer GM, Petrie IA, Boden RW, Dearling JL, Begent RH, Pedley RB (2005) Tumour parameters affected by combretastatin A-4 phosphate therapy in a human colorectal xenograft model in nude mice. Eur J Cancer 41(5): 799-806.

Fidarova EF, El-Emir E, Boxer GM, Qureshi U, Dearling JL, Robson MP, Begent RH, Trott KR, Pedley RB (2008) Microdistribution of targeted, fluorescently labeled anti-carcinoembryonic antigen antibody in metastatic colorectal cancer: implications for radioimmunotherapy. Clin Cancer Res 14(9): 2639-2646.

Folarin AA, Konerding MA, Timonen J, Nagl S, Pedley RB (2010) Three-dimensional analysis of tumour vascular corrosion casts using stereoimaging and micro-computed tomography. Microvasc Res 80(1): 89-98.

Hadimani MB, MacDonough MT, Ghatak A, Strecker TE, Lopez R, Sriram M, Nguyen BL, Hall JJ, Kessler RJ, Shirali AR, Liu L, Garner CM, Pettit GR, Hamel E, Chaplin DJ, Mason RP, Trawick ML, Pinney KG (2013) Synthesis of a 2-aryl-3-aroyl indole salt (OXi8007) resembling combretastatin A-4 with application as a vascular disrupting agent. J Nat Prod 76(9): 1668-1678.

Hanahan D, Weinberg RA (2011) Hallmarks of cancer: the next generation. Cell 144(5): 646-674.

Kalber TL, Waterton JC, Griffiths JR, Ryan AJ, Robinson SP (2008) Longitudinal in vivo susceptibility contrast MRI measurements of LS174T colorectal liver metastasis in nude mice. J Magn Reson Imaging 28(6): 1451-1458.

Koh DM, Blackledge M, Collins D, Padhani A, Wallace T, Wilton B, Taylor NJ, Stirling JJ, Sinha R, Walicke P, Leach M, Judson I, Nathan P (2009) Reproducibility and changes in the apparent diffusion coefficients of solid tumours treated with combretastatin A4 phosphate and bevacizumab in a two-centre phase I clinical trial. Eur Radiol 19(11): 2728-2738.

Landuyt W, Verdoes O, Darius DO, Drijkoningen M, Nuyts S, Theys J, Stockx L, Wynendaele W, Fowler JF, Maleux G, Van den Bogaert W, Anne J, van OA, Lambin P (2000) Vascular targeting of solid tumours: a major 'inverse' volume-response relationship following combretastatin A-4 phosphate treatment of rat rhabdomyosarcomas. Eur J Cancer 36(14): 1833-1843.

Lee JA, Biel NM, Kozikowski RT, Siemann DW, Sorg BS (2014) In vivo spectral and fluorescence microscopy comparison of microvascular function after treatment with OXi4503, Sunitinib and their combination in Caki-2 tumors. Biomed Opt Express 5(6): 1965-1979.

Liu L, Mason RP, Gimi B (2015) Dynamic bioluminescence and fluorescence imaging of the effects of the antivascular agent Combretastatin-A4P (CA4P) on brain tumor xenografts. Cancer Lett 356(2 Pt B): 462-469.

Mason RP, Zhao D, Liu L, Trawick ML, Pinney KG (2011) A perspective on vascular disrupting agents that interact with tubulin: preclinical tumor imaging and biological assessment. Integr Biol (Camb) 3(4): 375-387.

Nathan P, Zweifel M, Padhani AR, Koh DM, Ng M, Collins DJ, Harris A, Carden C, Smythe J, Fisher N, Taylor NJ, Stirling JJ, Lu SP, Leach MO, Rustin GJ, Judson I (2012) Phase I trial of combretastatin A4 phosphate (CA4P) in combination with bevacizumab in patients with advanced cancer. Clin Cancer Res 18(12): 3428-3439.

Nielsen T, Bentzen L, Pedersen M, Tramm T, Rijken PF, Bussink J, Horsman MR, Ostergaard L (2012) Combretastatin A-4 phosphate affects tumor vessel volume and size distribution as assessed using MRI-based vessel size imaging. Clin Cancer Res 18(23): 6469-6477.

Patterson DM, Zweifel M, Middleton MR, Price PM, Folkes LK, Stratford MR, Ross P, Halford S, Peters J, Balkissoon J, Chaplin DJ, Padhani AR, Rustin GJ (2012a) Phase I clinical and pharmacokinetic evaluation of the vascular-disrupting agent OXi4503 in patients with advanced solid tumors. Clin Cancer Res 18(5): 1415-1425.

Patterson DM, Zweifel M, Middleton MR, Price PM, Folkes LK, Stratford MRL, Ross P, Halford S, Peters J, Balkissoon J, Chaplin DJ, Padhani AR, Rustin GJS (2012b) Phase I clinical and pharmacokinetic evaluation of the vascular-disrupting agent OXi4503 in patients with advanced solid tumors. Clin Cancer Res 18(5): 1415-1425.

Pedley RB, El-Emir E, Flynn AA, Boxer GM, Dearling J, Raleigh JA, Hill SA, Stuart S, Motha R, Begent RH (2002) Synergy between vascular targeting agents and antibody-directed therapy. Int J Radiat Oncol Biol Phys 54(5): 1524-1531.

Ramasawmy R, Campbell-Washburn AE, Wells JA, Johnson SP, Pedley RB, Walker-Samuel S, Lythgoe MF (2014) Hepatic arterial spin labelling MRI: an initial evaluation in mice. NMR Biomed 28(2): 272-280.

Rice GC, Leiberman DP, Mathie RT, Ryan CJ, Harper AM, Blumgart LH (1977) Liver tissue blood flow measured by $85 \mathrm{Kr}$ clearance in the anaesthetized rat before and after partial hepatectomy. Br J Exp Pathol 58(3): 243-250.

Robinson SP, Kalber TL, Howe FA, McIntyre DJ, Griffiths JR, Blakey DC, Whittaker L, Ryan AJ, Waterton JC (2005) Acute tumor response to ZD6126 assessed by intrinsic susceptibility magnetic resonance imaging. Neoplasia 7(5): 466-474.

Salmon HW, Siemann DW (2006) Effect of the second-generation vascular disrupting agent OXi4503 on tumor vascularity. Clin Cancer Res 12(13): 4090-4094.

Schalkx HJ, van SM, Coenegrachts K, van den Bosch MA, van Kessel CS, van HR, van Erpecum KJ, Verkooijen HM, Pluim JP, Veldhuis WB, van Leeuwen MS (2014) Liver perfusion in dynamic contrast-enhanced magnetic resonance imaging (DCE-MRI): comparison of enhancement in Gd-BT-DO3A and Gd-EOB-DTPA in normal liver parenchyma. Eur Radiol 24(9): 2146-2156.

Siemann DW (2011) The unique characteristics of tumor vasculature and preclinical evidence for its selective disruption by tumor-vascular disrupting agents. Cancer Treat Rev 37(1): 63-74.

Walker-Samuel S, Orton M, McPhail LD, Boult JK, Box G, Eccles SA, Robinson SP (2010) Bayesian estimation of changes in transverse relaxation rates. Magn Reson Med 64(3): 914-921.

Wankhede M, Dedeugd C, Siemann DW, Sorg BS (2010) In vivo functional differences in microvascular response of $4 \mathrm{~T} 1$ and Caki-1 tumors after treatment with OXi4503. Oncol Rep 23(3): 685-692.

Workman P, Aboagye EO, Balkwill F, Balmain A, Bruder G, Chaplin DJ, Double JA, Everitt J, Farningham DA, Glennie MJ, Kelland LR, Robinson V, Stratford IJ, Tozer GM, Watson S, Wedge SR, Eccles SA (2010) Guidelines for the welfare and use of animals in cancer research. Br J Cancer 102(11): 1555-1577.

Zou JX, Wang MJ, Lei XJ, Chen XG (2014) 3.0 T MRI arterial spin labeling and magnetic resonance spectroscopy technology in the application of Alzheimer's disease. Exp Gerontol 60: 31-36.

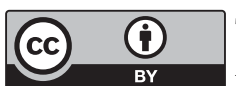

This work is licensed under the Creative Commons Attribution 4.0 International License. To view a copy of this license, visit http://creativecommons.org/licenses/by/4.0/ 